\title{
Single-particle identification of trace arsenic constituents in environmental samples
}

\author{
V.S.T. Ciminelli ${ }^{1,2}$, I.D. Delbem ${ }^{2,3}$, E. Freitas ${ }^{2,3}$, M. Morais ${ }^{4}$ \& M. Gasparon ${ }^{2,5}$ \\ ${ }^{1}$ Universidade Federal de Minas Gerais-UFMG, Department of Metallurgical and Materials Engineering, \\ Belo Horizonte, Brazil \\ ${ }^{2}$ National Institute of Science and Technology on Mineral Resources, Water and Biodiversity, INCT-Acqua, Brazil \\ ${ }^{3}$ Universidade Federal de Minas Gerais-UFMG, Center of Microscopy, Belo Horizonte, Brazil \\ ${ }^{4}$ Kinross Brasil Mineração, Brazil \\ ${ }^{5}$ The University of Queensland, School of Earth and Environmental Sciences, St Lucia, Australia
}

\begin{abstract}
An analytical protocol was developed to identify arsenic (As) in soil samples and in the $\leq 10 \mu \mathrm{m}$ fraction of surface dust samples (fine surface dust-FSD). Single-particle identification of trace As constituents was undertaken by combining scanning electron microscopy with automated image analyses and high-resolution, transmission electron microscopy. Two forms of As association with iron and aluminum nanoscale phases were identified. In the predominant one, As was identified in oriented aggregates formed by crystalline nanoparticles of Fe-(hydr)oxides. In the FSD samples, As was additionally detected in an assembly of hematite and goethite nanocrystals forming larger particles of few hundreds of nanometers, often entangled with phyllosilicates. These mixed phases carried various elements, such as $\mathrm{P}, \mathrm{Ba}, \mathrm{Pb}$, among others. Even rare As-bearing phases (e.g., 1 to 9 particles out of approx. 30,000 particles analyzed), such as arsenopyrite and ferric arsenate, and possibly scorodite were identified in some samples. The developed analytical protocol brings a novel and practical contribution to As speciation in environmental samples.
\end{abstract}

\section{INTRODUCTION}

Precise, single particle characterization of arsenic (As)-bearing phases in environmental samples is not a simple task despite the advances in analytical techniques. Bulk X-ray absorption spectroscopy has been applied to identify the molecular environment of As in various matrices for more than two decades (Foster et al., 1998). Theoretical modeling combined with spectroscopic techniques has further advanced the understanding of the mechanisms of As fixation in the environment (Ladeira et al., 2001). Micro-Xray fluorescence combined with microfocused-X-ray absorption spectroscopy has enabled in situ characterization of As with spatial resolution usually down to the micrometer level (Ono et al., 2015). Nevertheless, none of the aforementioned methods provides the spatial resolution necessary to investigate highly heterogeneous nanoscale phases in environmental samples, down to a few nanometres, or allow statistically sound quantification of As-bearing phases.

To overcome these limitations, our group has combined scanning electron microscopy with automated image analysis and high-resolution transmission electron microscopy (HRTEM). An analytical protocol has been developed and applied to soil and fine $(\leq 10 \mu \mathrm{m}$ fraction) surface dust samples collected in a gold mining region. The results will demonstrate that the developed analytical protocol brings a novel and practical contribution to As speciation in environmental samples.

\section{METHODS/EXPERIMENTAL}

\subsection{Sampling, sample preparation and analysis}

The collection of surface soil $(0-20 \mathrm{~cm})$ samples was undertaken following local and international protocols (USEPA, 1991) in four geological units and four classes of soils, comprising areas of gold mineralization and areas representing the region's background. The samples were collected in areas with no indication of anthropogenic activities. The bulk samples were oven-dried at $40^{\circ} \mathrm{C}$ for 12 hours then disaggregated, split into sub-samples and sieved at $2 \mathrm{~mm}$, and then finely-ground $(<44 \mu \mathrm{m})$ for chemical analyses and particle characterization by transmission electron microscopy TEM.

Surface dust sampling was conducted in the residential area (where citizens may be regularly exposed to resuspended dust) near the gold mine operation in two campaigns (dry and wet seasons). These samples possibly represent a combination of fugitive dust from construction and excavation sites (including the mine site), natural geological background and baseline 
associated with local and regional industrial and agricultural activities. The collection and storage followed the procedures described by USEPA (1991). Aiming to assess the respiratory exposure, five surface dust samples were sieved to obtain the $\leq 10 \mu \mathrm{m}$ fraction (Fine surface dust - FSD). Two samples collected from the crushing area within the mine site were also sieved at $10 \mu \mathrm{m}$. The sieving apparatus consisted of adapting an Ultrasonic Sieving (HK Technologies, USA) to a mechanical vibrator (Cleveland Vibrator Company Model: VJ-1212, USA).

The acid extractable As content in the soil samples was determined following digestion with aqua regia using a microwave-assisted (Ethos, Milestone) digestion procedure (USEPA, 2007). Arsenic was analyzed by inductively coupled plasma optical emission spectrometry (ICP-OES) (Perkin Elmer Optima 7300DV) or ICP-MS (Agilent $7500 \mathrm{cs}, \mathrm{CA}$, USA). Two standard reference materials (NIST SRM 2710a and CANMET/ CCRMP-Till-3) were analyzed together with each batch of 10 soil samples. Duplicates and analytical blanks were analyzed as well. Arsenic recoveries ranged from 84 to $101 \%$. All blank extractions returned values below the method detection limits.

\subsection{Electron Microscopy analyses}

The characterization of As-bearing phases and quantitative mineralogy were based on single particle analyses using a FEI Quanta 650 Field Emission Gun Scanning Electron Microscope (FEG-SEM) equipped with two Bruker Quantax X-Flash 5010 energy dispersion X-ray (EDX) detectors and FEI's Mineral Liberation Analyzer-MLA for data acquisition and process. The grain-based X-ray mapping (GXMAP) measurement mode was applied to the analyses of polished sections and loose particles. In this mode, a series of backscattered electron (BSE) images is collected. Identification of mineral grains by MLA is based on BSE image segmentation and collection of EDX-spectra of the particles/grains. Collected EDXspectra are then classified using a pre-defined list of mineral spectra collected by the user. The method has a resolution of grain size down to $0.1-0.2 \mu \mathrm{m}$ (Gu et al., 2003). For the TEM analyses each powder sample was dispersed in Milli-Q water in Eppendorf tubes and sonicated in ultrasound bath. A drop of each suspension was placed on carbon coated $\mathrm{Cu}$-TEM grids $(300$ mesh) and left to dry in a desiccator. The analysis was performed using High Resolution TEM (HRTEM), Scanning TEM (STEM), EDX spectroscopy and Electron Energy-Loss Spectroscopy (EELS) using a FEI TEM Tecnai G2-20 (200 kV).

\section{RESULTS AND DISCUSSION}

\subsection{As-bearing phases}

Table 1 shows the main mineral phases in the soil samples according to the analyses carried out by
Table 1. Main mineral phases (\%) and arsenic minerals (number of particles) identified by MLA in three soil samples.

\begin{tabular}{|c|c|c|c|c|}
\hline \multirow[b]{2}{*}{ Samples } & \multirow{2}{*}{$\begin{array}{l}\text { S0 } \\
<2 \mathrm{~mm}\end{array}$} & \multirow{2}{*}{$\begin{array}{l}\mathrm{S} 1 \\
<2 \mathrm{~mm}\end{array}$} & \multicolumn{2}{|l|}{ S4 } \\
\hline & & & $>2 \mathrm{~mm}$ & $<2 \mathrm{~mm}$ \\
\hline Quartz & 21.6 & 49.1 & 26.6 & 28.2 \\
\hline Other silicates & 72.1 & 39.8 & 27.6 & 55.1 \\
\hline FeOx-As free & 1.7 & 1.4 & 22.6 & 6.7 \\
\hline FeOx-As & 0.6 & 4.1 & 21.3 & 4.4 \\
\hline Ilmenite & n.d. & 2.7 & n.d. & 4.2 \\
\hline Others $(<2 \% \mathrm{wt})$ & 4.0 & 2.9 & 1.9 & 1.4 \\
\hline \multicolumn{5}{|c|}{ Number of particles } \\
\hline Arsenopyrite & n.d. & 4 & n.d. & n.d. \\
\hline Scorodite & n.d. & 1 & n.d. & n.d \\
\hline $\mathrm{FeOx}-\mathrm{As}$ & 738 & 2,901 & 11,702 & 1,968 \\
\hline Total particles & 79,330 & 41,831 & 33,864 & 59,477 \\
\hline $\operatorname{As}\left(\mathrm{mg} \mathrm{kg}^{-1}\right)$ & 411 & 1,560 & 7,556 & 1,355 \\
\hline
\end{tabular}

n.d. $=$ not detected.

the MLA. The main phases (>2 wt.\%) are the silicates: quartz $\left(\mathrm{SiO}_{2}\right)$, mica/clay minerals and microcline $\left(\mathrm{KAlSi}_{3} \mathrm{O}_{8}\right)$ and others. Goethite $(\mathrm{FeO}(\mathrm{OH}))$ and hematite $\left(\alpha-\mathrm{Fe}_{2} \mathrm{O}_{3}\right)$ are the main $\mathrm{Fe}$-(hydr)oxides. The MLA tool allows for quantitative single particle analysis of a large number of grains. The total number of particles analyzed per sample ranged from 33,864 to 79,330 , thus providing good statistics. The differences in the number of particles reflect differences in particle size distribution for a fixed measuring time. Large variations in the content of Fe-(hydr)oxides from $2 \%$ to $44 \%$ are observed. In general, the As concentration increases with the increase of the concentration of Fe-(hydr)oxides, with a significant As-enrichment in the coarse fraction.

Arsenic is found mainly associated with $\mathrm{Fe}$ (hydr)oxides, with rare arsenopyrite and ferric arsenate, likely scorodite. Arsenopyrite and scorodite are the main As phases in the local sulfide and oxidized ore bodies, respectively. The relatively low number arsenopyrite particles and other sulfides (not shown) is consistent with the low bulk sulfur concentration (range of $<100$ to $288 \mathrm{mg} \mathrm{kg}^{-1}$ ). The results indicate a small contribution of sulfides and arsenates from the mineralized lithologies to the bulk soil chemistry. The quantification of the mineral phases determined by the MLA was shown to be consistent with the chemical analyses (not shown) of the major elements (iron and silicon) within $15 \%$ variation.

A BSE-SEM image of polished sections prepared from the soil samples show typical mineral associations found in the soil samples: quartz inclusion and the intergrowth of phyllosilicate lamellae (muscovite) with Fe-(hydr)oxides (goethite or hematite) (Fig. 1).

The MLA was also employed to analyze the mineral phases in the FSD samples. The method allowed a good reading of particles with diameter smaller than $10 \mu \mathrm{m}$. The main constituents were again the silicate 
Table 2. Main mineral phases (\%) and As minerals (no. particles) identified by MLA in four FSD samples.

\begin{tabular}{|c|c|c|c|c|}
\hline Samples & $\begin{array}{l}\mathrm{FC} \\
<2 \mathrm{~mm}\end{array}$ & $\begin{array}{l}\mathrm{F} 2 \\
<2 \mathrm{~mm}\end{array}$ & $\begin{array}{l}\mathrm{F} 17 \\
>2 \mathrm{~mm}\end{array}$ & $\begin{array}{l}\mathrm{F} 19 \\
<2 \mathrm{mr}\end{array}$ \\
\hline Mica/Clay & 85.0 & 66.0 & 58.0 & 56.6 \\
\hline Quartz & 9.7 & 18.2 & 7.9 & 12.0 \\
\hline $\mathrm{Fe}-\mathrm{Ox}$ & 1.6 & 3.8 & 4.9 & 9.0 \\
\hline Organic matter & 0.4 & 2.4 & 4.9 & 4.0 \\
\hline Other silicates & 1.8 & 4.5 & 14.2 & 10.0 \\
\hline Carbonates & 1.4 & 2.7 & 7.3 & 6.0 \\
\hline Others & 0.1 & 2.4 & 2.8 & 2.4 \\
\hline \multicolumn{5}{|c|}{ Number of particles } \\
\hline Arsenopyrite & 8 & 1 & n.d. & 1 \\
\hline Scorodite & 9 & 1 & 3 & 2 \\
\hline $\mathrm{FeOx}-\mathrm{As}$ & 109 & 240 & 150 & 211 \\
\hline As mixed phases & 47 & 17 & 60 & 32 \\
\hline Tot. no. particles & 35,624 & 36,856 & 35,144 & 38,517 \\
\hline As $\left(\mathrm{mg} \mathrm{kg}^{-1}\right)$ & 279 & 445 & 212 & 265 \\
\hline
\end{tabular}

n.d. $=$ not detected

minerals, such as quartz, muscovite and other clay minerals; Fe oxy-hydroxides; carbonates and organic matter - identified by the typical morphology and the level of carbon - associated with low concentrations of elements such as $\mathrm{Si}, \mathrm{Al}$ and $\mathrm{Mg}$.

In the FSD samples As is found in five phases: (i) arsenopyrite, (ii) scorodite (iii) iron oxy-hydroxide, (iv) mixed phases and (v) muscovite/clay. Iron oxyhydroxides were identified as the major As-bearing phase in all the samples followed by the As-bearing mixed phases. According to the EDX analyses, these mixed phases contain $\mathrm{Al}$ (2.44 to $47.31 \%)$, Si (1 to $4.02 \%), \mathrm{P}(0.47$ to $34.29 \%)$, Ca $(0.47$ to $7.34 \%), \mathrm{Fe}$ $(0.18$ to $23.60 \%)$, As $(0.06$ to $7.23 \%), \mathrm{Ba}(0.11$ to $6.01 \%), \mathrm{Pb}(0.14$ to $25.64 \%), \mathrm{O}(50.67$ to $78.80 \%)$, whereas $\mathrm{Na}, \mathrm{Ti}, \mathrm{Cl}, \mathrm{S}, \mathrm{K}, \mathrm{V}, \mathrm{Cr}, \mathrm{Cu}, \mathrm{Sr}, \mathrm{Cd}$ appear as minors. No specific known mineral phase could be assigned to these phases. Arsenopyrite and scorodite are rare. In the case of FSD samples, the preparation of polished sections for quantitative MLA analyses is not possible due to the fine particle size of the material and though the numbers could not be fully validated by chemical analyses, there is a clear and consistent trend when comparing both methods. The technique allows for the identification of As-bearing phases from a significant population of particulate material (up to 30,000 particles), in a heterogenous, complex mineral assembly, which would not have been possible by the common techniques applied to particulate analyses. The refinement of the method for quantitative analyses of FSD is under development.

The main As carriers - aggregates and mixed phases - were further investigated by TEM analysis. Based on $\mathrm{d}_{\mathrm{hkl}}$ spaces, the aggregates (present in both soil and FSD samples) were identified as goethite and hematite. Freitas et al. (2015) investigated As- enriched Fe-Al-oxisols after their use as liners in disposal facilities of sulfide tailings. The

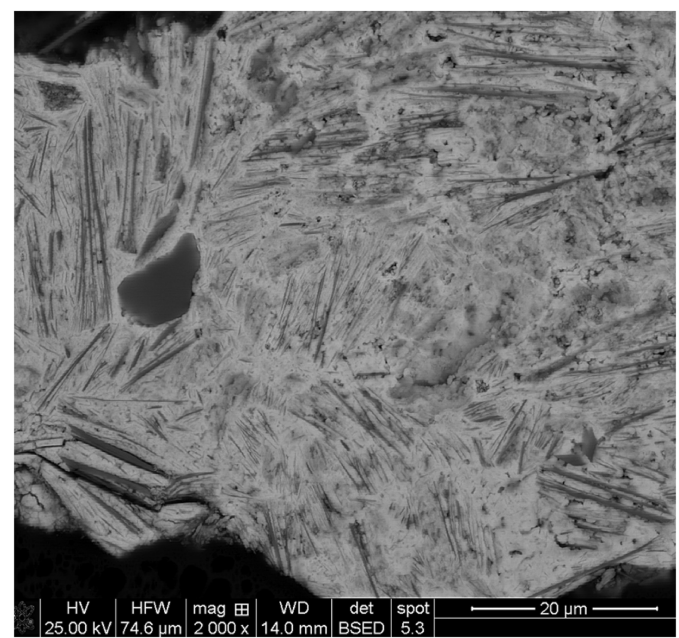

Figure 1. BSE-SEM images of polished sections showing the inclusion of quartz and intergrowth of phyllosilicate lamellae (muscovite) with Fe-(hydr)oxides (goethite) in soil samples.

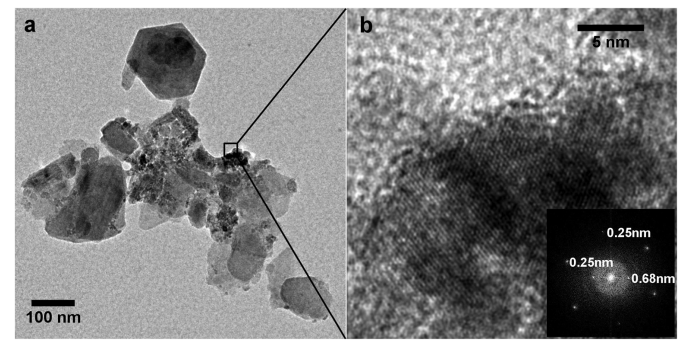

Figure 2. Phyllosilicates entangled with mixed phases in FSD samples (left), and a HRTEM image of the oriented aggregate of nanoparticles (right).

results demonstrated that As was present in oriented aggregates formed by crystalline nanoparticles of Fe-(hydr)oxides. The same pattern was found in the soil samples collected in the present study. In addition to oriented aggregates described before, TEM analyses showed that the mixed phases are essentially an assembly of hematite and goethite nanocrystals that form larger particles of a few hundreds of nanometers. These mineral phases were identified by HRTEM image analysis and selected area diffraction- SAD. Aluminum was also detected.

The nanoparticles of Fe-(hydr)oxides are often entangled with phyllosilicates (Figure 2). The TEMEDS analysis has not shown the presence of other elements apart from $\mathrm{O}, \mathrm{Fe}, \mathrm{Al}$, and As in probed particles. The occurrence of other elements such as $\mathrm{P}$ and Si observed in the SEM-EDS data might be due to the larger interaction volume (a few micrometers size), around the target particle, where the X-rays are emitted from thicker samples. 


\section{CONCLUSIONS}

An analytical protocol, combining scanning electron microscopy with automated image analysis and high-resolution transmission electron microscopy, was developed and applied to soil and fine $(\leq 10 \mu \mathrm{m}$ fraction) surface dust samples. This protocol allows for a statistically sound quantification of As-bearing phases with the spatial resolution necessary to investigate highly heterogeneous nanoscale phases in environmental samples, down to a few nanometres. Arsenic was mainly identified in oriented aggregates formed by crystalline nanoparticles of Fe-(hydr)oxides. In the FSD samples, As was additionally detected in an assembly of hematite and goethite nanocrystals forming larger particles of a few hundreds of nanometers, and often entangled with phyllosilicates. Rare arsenopyrite and scorodite were identified in the samples.

\section{ACKNOWLEDGEMENTS}

The authors are grateful to the Brazilian government agencies - CNPq, FAPEMIG and CAPES, and to Kinross Brasil Mineração (KBM) for financial support and the PVE fellowship from the Science Without Borders program to $\mathrm{M}$. Gasparon. The authors also acknowledge the Center of Microscopy/UFMG. Prof. Jack Ng and Dr. Lixia Qi at UQ are gratefully acknowledged for hosting M. Morais in the UQ Laboratory. The authors also thank Dr Claudia L. Caldeira, Patricia Lopes and Filipe A.T. Alves at UFMG for the
ICP-OES analyses, and Dr. Marcus Manoel Fernandes for the soil samples.

\section{REFERENCES}

Foster, A.L., Brown, Jr. G.E., Tingle, T.N. \& Parks, G.A. 1998. Quantitative As speciation in mine tailings using X-ray absorption spectroscopy. Am. Mineral. 83: 553-68.

Freitas, E.T.F., Montoro, L.A., Gasparon, M. \& Ciminelli, V.S.T. 2015. Natural attenuation of arsenic in the environment by immobilization in nanostructured hematite. Chemosphere 138: 340-347.

$\mathrm{Gu}, \mathrm{Y} .2003$. Automated scanning electron microscope based mineral liberation analysis. J. Miner. Mater. Character. Eng. 2: 33-41.

Ladeira, A.C.Q., Ciminelli, V.S.T., Alves, M.C.M. \& Duarte, H.A. 2001. Mechanism of anion retention from EXAFS and Density Functional Calculations: Arsenic (V) adsorbed on Gibbsite. Geochim. Cosmochim. Acta 65(8): 1211-1217.

Mason, B.J. 1992. Preparation of soil sampling protocols: sampling techniques and strategies (No. PB-92220532/XAB). Nevada Univ., Las Vegas, NV (United States). Environmental Research Center.

Ono, F.B., Tappero, R., Sparks, D. \& Guilherme, L.R.G. 2015. Investigation of arsenic species in tailings and windblown dust from a gold mining area. Environ. Sci. Poll. Res. 23(1): 638-647.

USEPA-Environmental Protection Agency. 1991. Compendum of ERT waste sampling procedures. Emergency Response Division. EPA/540/P-91/008. Oswer Directive 9360.4-07.

USEPA-United States Environmental Protection Agency 2007. Microwave assisted acid digestion of sediments, sludges, soils and oils - Method 3051a. Revision 1. 